\title{
Die Gesneriaceen Afrikas und ihre vogelblütigen Vertreter: Streptocarpus dunnii und S. myoporoides
}

\author{
Anton Weber, Dirk U. Bellstedt \& Michael Möller
}

\begin{abstract}
The African continent (incl. Madagascar and the Comoro Islands) is relatively poor in Gesneriaceae. Moreover, African Gesneriaceae are also poor in typical tropical pollination syndromes such as ornitho- or chiropterophily (bird or bat pollination). The latter is completely absent and there are only two species that clearly exhibit a bird pollination syndrome: Streptocarpus dunnii and S. myoporoides. These species are presented and discussed here in context with a new concept of Streptocarpus. Concluding from differences in vegetative, inflorescence and floral morphology (molecular, chromosomal etc. data are not yet available for $S$. myoporoides), the two species are apparently not closely related and it appears that ornithophily has evolved twice in the African Gesneriaceae.
\end{abstract}

\section{Zusammenfassung}

Der Afrikanische Kontinent (inkl. Madagaskar und die Komoren) ist relativ arm an Vertretern der Gesneriaceae. Afrikanische Gesneriaceen sind auch arm an typisch tropischen Bestäubungsmustern wie Vogel- und Fledermausblütigkeit. Während das letztere Syndrom völlig fehlt, gibt es nur zwei Arten, die klar das Syndrom der Vogelblütigkeit (Ornithophilie) aufweisen: Streptocarpus dunnii and S. myoporoides. Diese beiden Arten werden hier im Zusammenhang mit dem neuen taxonomischen Konzept der Gattung Streptocarpus näher vorgestellt. Auf Grund der gravierenden Unterschiede im Bau des Vegetationskörpers, der Blütenstände und Blüten (molekulare, chromosomale etc. Daten von S. myoporoides fehlen) kann man darauf schließen, dass die beiden Arten nicht nahe verwandt sind und die Ornithophilie bei den afrikanischen Gesneriaceen zweimal voneinander unabhängig entstanden ist.

\section{Einleitung}

Neben dem Usambaraveilchen (Streptocarpus ionanthus, früher Saintpaulia ionantha) und den "Gloxinien“" (Zuchtformen von Sinningia speciosa) haben sich in den letzten Jahrzehnten weitere Gesneriaceen als Zimmerpflanzen etabliert: Streptocarpus rexii, S. primulifolius und verwandte Arten, und davon ausgehend eine Unzahl von Hybriden und Zuchtformen. Die Blütenform des Wildtyps ist die einer relativ großen Trichterblüte mit schwach zweilippigem Saum, die Färbung hell-blau, mit dunklen Linien (Saftmale), die in den Kronenschlund hineinführen (Abb. 1a, b). Man könnte glauben, das sei die typische Blütenform der Gattung Streptocarpus. Dem ist aber nicht so, insbesondere dadurch, dass vor einigen Jahren die Gattung neu definiert wurde und alle afrikanischen Gesneriaceen (außer Epithema) Streptocarpus einverleibt wurden (NisHil et al. 2015), hat sich das Spektrum an Blütenformen enorm erweitert.

Obwohl Streptocarpus ein riesiges Verbreitungsgebiet einnimmt (das westliche tropische Afrika sowie das gesamte zentral- und südöstliche Afri- ka, Madagaskar und die Komoren, Abb. 2), also tropisch-subtropisch geprägt ist, fällt auf, dass die Blüten fast immer das Syndrom der Insektenblütigkeit (Entomophilie) aufweisen. Typisch tropische Bestäubungssyndrome, wie wir sie von mittel-und südamerikanischen und asiatischen Gesneriaceen kennen, fehlen: Fledermausbestäubung (Chiropterophilie) fehlt komplett, und nur zwei Arten weisen eindeutig das Syndrom der Vogelbestäubung auf, nämlich Streptocarpus dunnii und S. myoporoides. Hier werden diese beiden interessanten und höchst attraktiven Arten näher vorgestellt.

\section{Die Gesneriaceen des}

\section{afrikanischen Kontinents}

Die Gesneriaceae sind eine hauptsächlich tropischsubtropisch verbreitete Familie mit etwa 150 Gattungen und an die 3700 Arten. Grob die Hälfte entfallen auf Mittel- und Südamerika, die andere Hälfte auf Afrika, Zentral-, Ost-, Süd- und Südostasien und die Inselwelt des Malaiischen Archipels und des Pazifiks.

Obwohl der Afrikanische Kontinent (mit Madagaskar und kleineren Inselgruppen) riesen- 


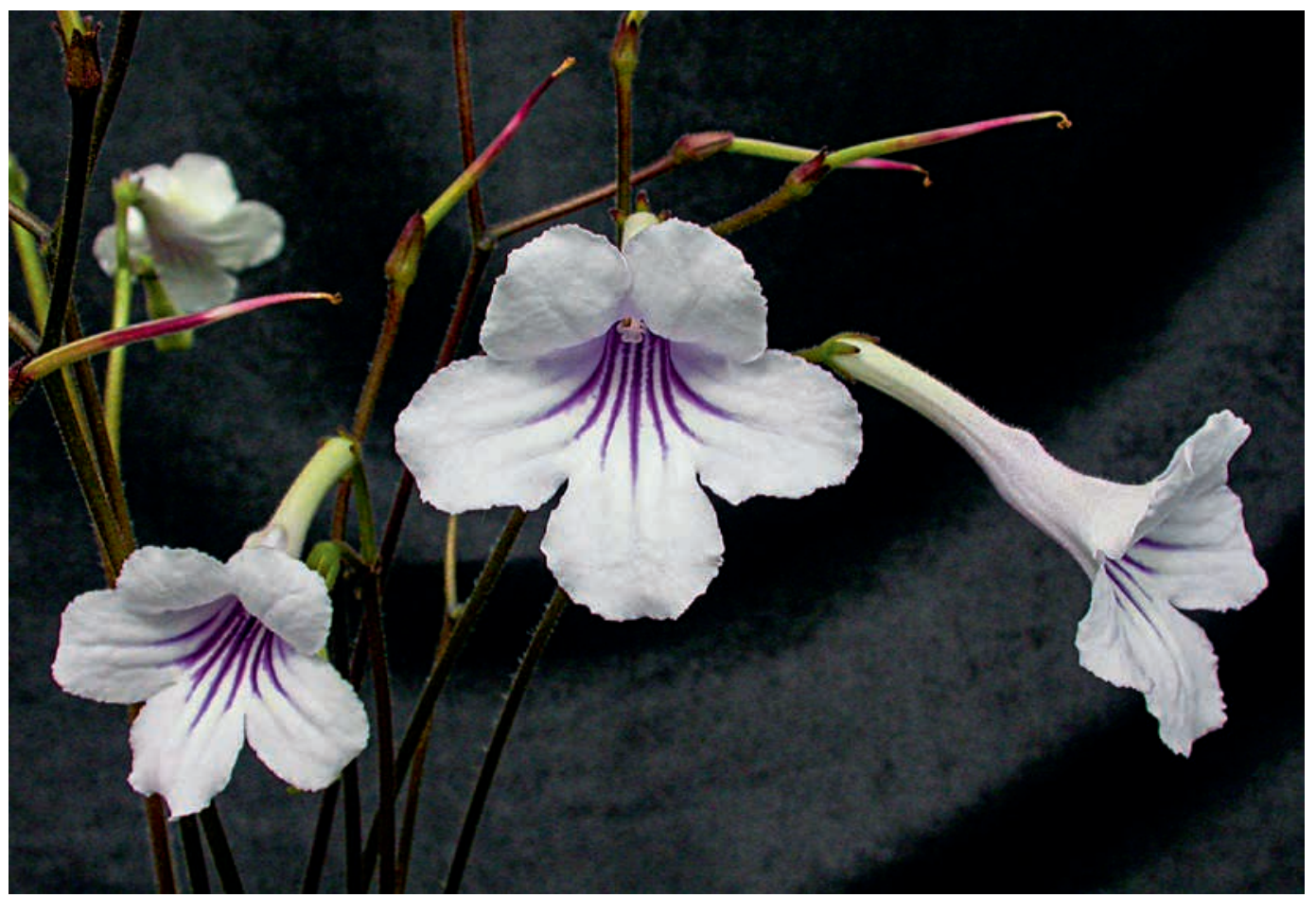

Abb. 1a: Blüten von Streptocarpus rexii. Diese und weitere Arten der Gattung Streptocarpus sind der Ursprung von zahlreichen Hybriden und Zuchtformen. (Foto: M. Möller)

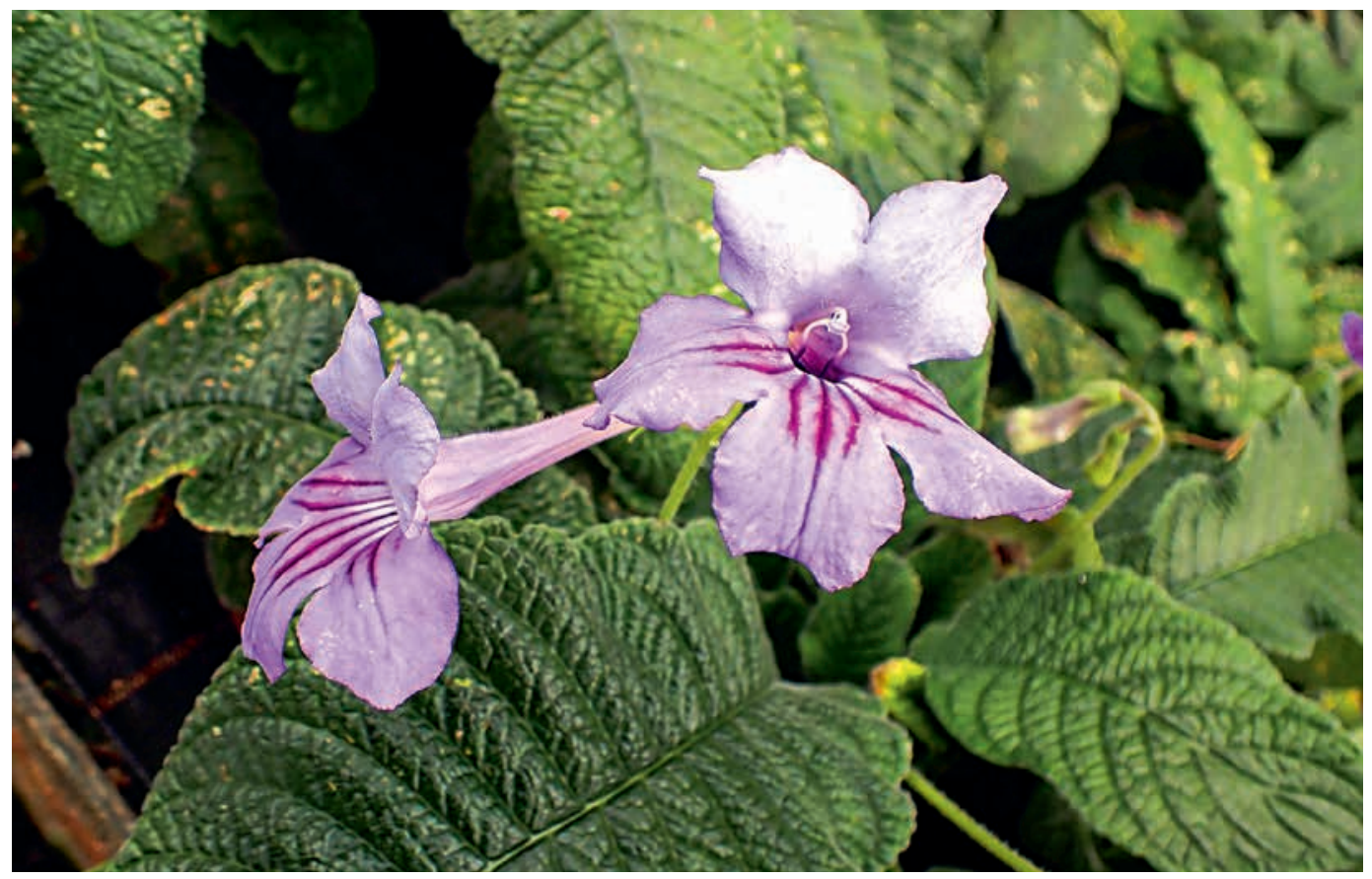

Abb. 1b: Streptocarpus primulifolius, eine weitere Ausgangsart für ornamentale Gesneriaceen. (Foto: M. MöLlER) 


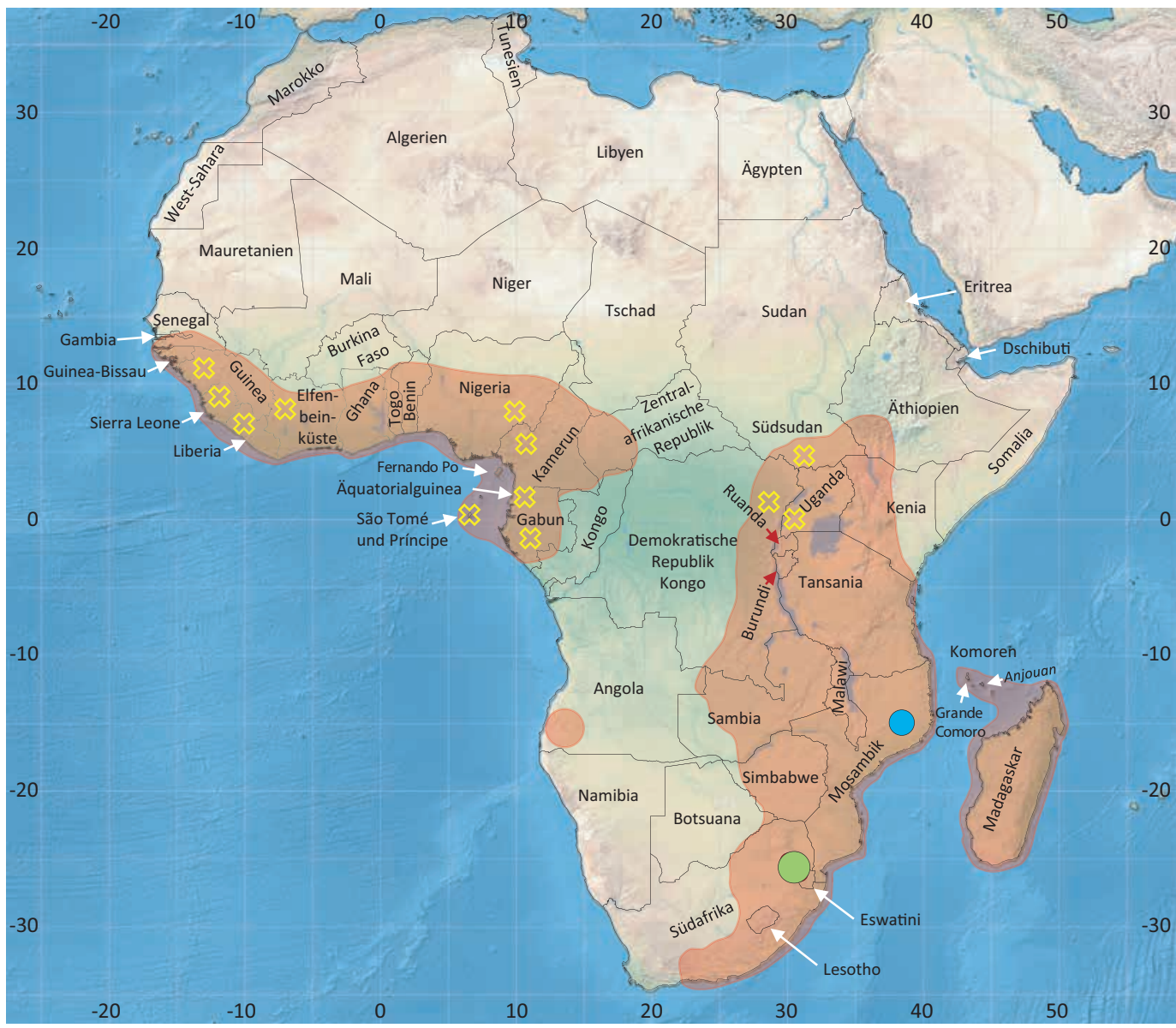

Abb. 2: Verbreitung der Gesneriaceen auf dem afrikanischen Kontinent. Rötlich-schattierte Gebiete: Streptocarpus. Gelbe Kreuze: Länder mit Fundorten von Epithema tenue. Grüner Kreis: Streptocarpus dunnii (nur in der Provinz Mpumalanga der Republik Südafrika und in den angrenzenden Teilen des Königreiches Eswatini (Swasiland). Blauer Kreis: S. myoporoides (endemisch im Mt. Ribáuè und Mt. Chinga Gebirge, Provinz Nampula, Mosambik). (Karte: erstellt mit SimpleMappr, Shorthouse 2010, von M. MöLler)

groß ist, sind hier bisher nur 180 Arten bekannt. Davon entfallen eine Art auf die Gattung Epithema (E. tenue), 132 Streptocarpus-Arten auf Afrika und 47 Streptocarpus-Arten auf Madagaskar und die Komoren - insgesamt eine vergleichsweise geringe Zahl. Das geflügelte Wort "Africa - odd man out" (Artenarmut der afrikanischen Regenwälder) trifft somit auch für die Gesneriaceen zu.

Epithema tenue kommt nur im nördlichen Teil des Verbreitungsgebietes von Streptocarpus vor, das in vier verschieden große Teilareale zerfällt (vgl. Abb. 2).

\section{Alte und neue taxonomische Konzepte der afrikanischen Gesneriaceen}

Traditionell hat man die afrikanischen Gesneriaceen in 10 Gattungen gegliedert. Die wohl bekannteste ist Saintpaulia zusammen mit der Hauptgattung Streptocarpus mit sich postfloral spiralig eindrehenden Kapselfrüchten (vgl. Abb. 3). Dazu kommen acht weitere, nur eine bis wenige Arten umfassende Gattungen mit geraden Früchten. Die molekularsystematischen Studien von Nishir et al. (2015) haben jedoch gezeigt, dass außer Epithema alle Gattungen (also auch Saintpaulia) in Streptocarpus einbezogen werden müssen. Die Drehung der Früchte erscheint als 
ursprüngliches Merkmal der Gattung. Sie ging in der Evolution mehrmals verloren: fünf $\mathrm{Mal}$ in der Untergattung Streptocarpella und drei Mal in der Untergattung Streptocarpus.

Taxonomisch nicht davon betroffen ist, wie erwähnt, die Gattung Epithema mit insgesamt 20 Arten (Bransgrove \& Middleton 2015). Sie hat ihre Hauptverbreitung in Südostasien und ist in Afrika mit E. tenue vertreten. Die Frage, wie Epithema bis nach Westafrika gekommen ist (Fernverbreitung?) oder ob das Verbreitungsgebiet der Gattung Epithema früher viel größer war als heute, ist zur Zeit ungeklärt. Streptocarpus hat sich in Afrika anscheinend von Norden her nach Süden und Westen ausgebreitet (Möller \& Cronk 2001).

\section{Taxonomische Geschichte von Streptocarpus}

Die Gattung Streptocarpus wurde von dem Britischen Botaniker John Lindley (1799-1865) im Jahre 1828 etabliert, die begleitende Illustration ist in Abb. 3. wiedergegeben. Sie zeigt sehr schön die Blütenform, die blasslila Blütenfarbe mit den linienförmigen Saftmalen und eine gedrehte Frucht. Die beiden Fruchtklappen drehen sich im Laufe der Fruchtentwicklung in Form einer Doppelhelix ein.

LINDLEY stellte die Gattung Streptocarpus nicht auf Grund einer neuentdeckten Art auf, sondern auf der Basis einer Pflanze, die schon vorher als Didymocarpus rexii beschrieben worden war. Exemplare dieser Pflanze und Samen wurden von J. Bowie (1789-1869) in Südafrika gesammelt und nach Kew geschickt. Die formale Beschreibung als neue Art (Didymocarpus rexii) erfolgte 1827 durch W. J. Hooker (1785-1865). Streptocarpus rexii ist die Typus-Art von Streptocarpus und heißt mit vollem Namen Streptocarpus rexii (Bowie ex Hook.) Lindl.

Im Laufe der Zeit sind mehr als 130 Arten dazugekommen. Die erste zusammenfassende und umfassende Darstellung von Streptocarpus (im traditionellen Sinne, 132 Arten) findet sich im Buch "Streptocarpus. An African Plant Study" von Hilliard \& Burtt (1971). Diese

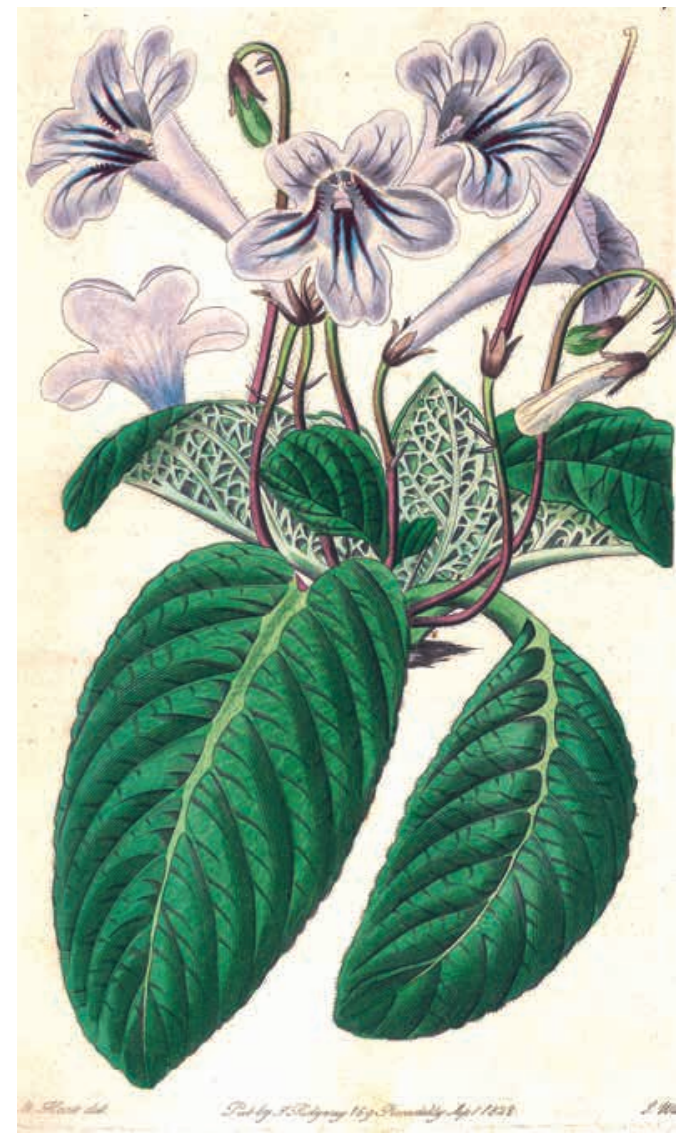

Abb. 3: Streptocarpus rexii, die Typus-Art der Gattung Streptocarpus. Die Abbildung ist Teil der Erstbeschreibung der Gattung Streptocarpus. (Lindley 1828, T. 1173)

enthält neben allgemeinen Kapiteln und Beschreibungen der bis dahin bekannten Arten zahlreiche instruktive Blütenzeichnungen, Fotos und Verbreitungskarten.

Nach 2000 wurde zuerst Linnaeopsis (DARBYSHIRE 2006) und später Saintpaulia (ChristenHusz 2012) auf der Basis der Daten von Möller \& CRONK (1997a,b, 1999) in Streptocarpus einbezogen, bis NisHir et al. (2015) alle afrikanisch-madagassischen Gesneriaceen (bis auf Epithema tenue) in Streptocarpus eingegliedert haben (siehe oben).

\section{Blütenvielfalt und Bestäubungssyndrome von Streptocarpus}

Nicht überraschend ist, dass sich, insbesondere auch durch die Einbeziehung der geradfrüchti- 


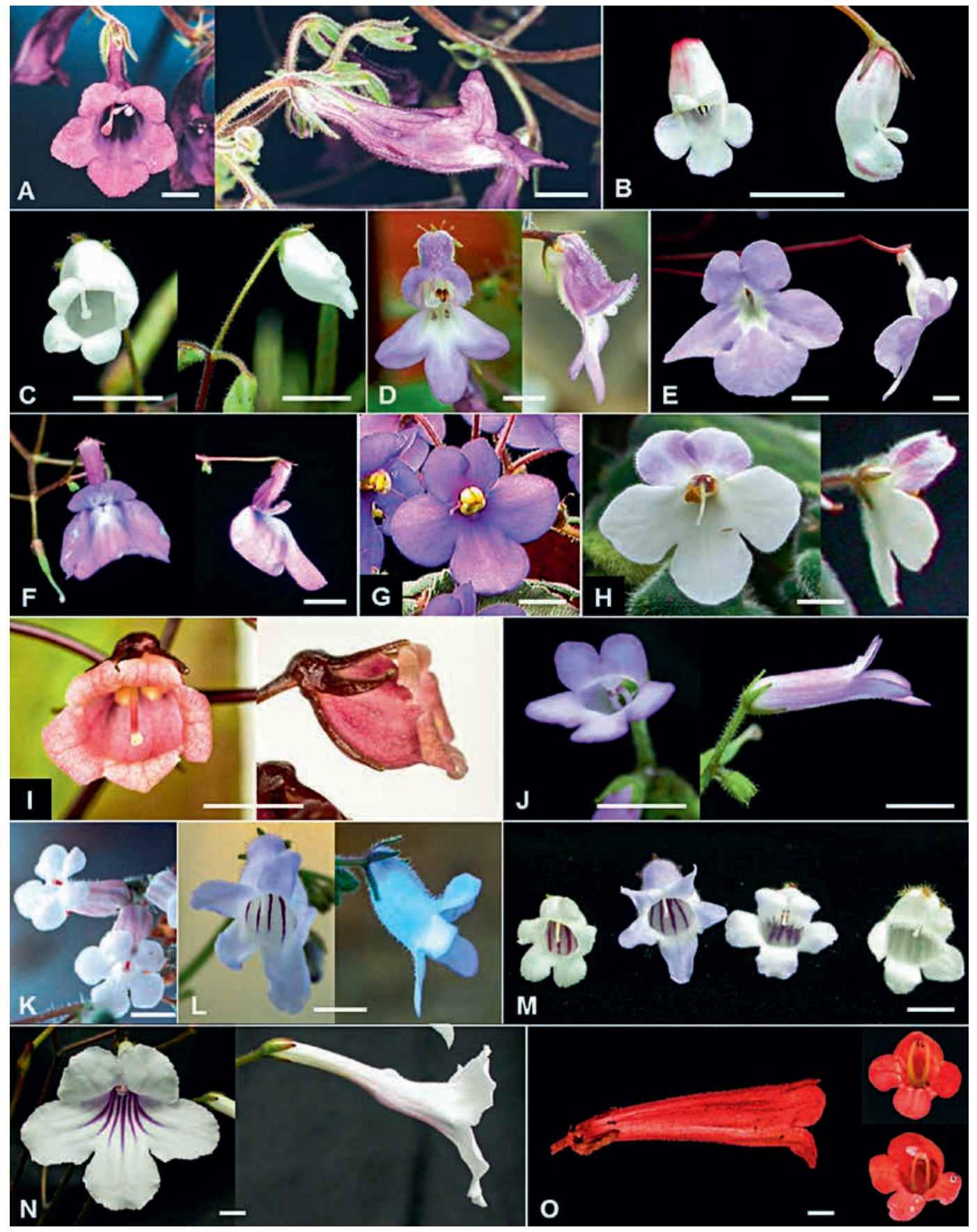

Abb. 4: Blütenformen (in Vorder- und Seitenansicht) in der Gattung Streptocarpus (sensu Nishir et al. 2015); A: S. nobilis (runde offene Blütenröhre); B: S. beampingaratrensis (kleine bauchige Blüte); C: S. muscosus (kleine bauchige Blüte); D: S. thysanotus (Labellanthus-Typ); E: S. saxorum („Schlüsselloch-Blüte“); F: S. caulescens (Maskenbüte); G: S. ionanthus (Saintpaulia-Typ); H: S. goetzeanus (Saintpaulia-Typ); I: S. papangae (kleine bauchige Blüte); J: S. hildebrandtii (kleine bauchige Blüte); K: S. ibityensis (kleine bauchige Blüte); L: S. montanus; M: (von links nach rechts): S. bullatus, S. montanus, S. parensis, S. schliebenii; N: S. rexii (runde offene Blütenröhre); O: $S$. dunnii (ornithophiler Typ). - Maßstab: 5 mm. Aus Nishi et al. (2015), Fig. 6. (Fotos: M. Möller) 
gen Gattungen, eine große Blütenvielfalt ergibt (Abb. 4). Harrison et al. (1999) haben versucht, die Blütenvielfalt von Streptocarpus (s. s.) zu gliedern und haben sechs Grundtypen unterschieden. Zuletzt haben MöLler et al. (2019) einen Versuch unternommen, die Blütenvielfalt und Bestäubungssyndrome von Streptocarpus nun in dem neu definierten Umfang zu ordnen. Sie unterscheiden sieben Haupt- und mehrere Subtypen. Der phylogenetisch ursprünglichste Typ ist nach den Studien von Hughes et al. (2006) jener, wie er bei $S$. beampingaratrensis oder $S$. muscosus anzutreffen ist (Abb. $4 \mathrm{~B}, \mathrm{C}$ ). Die Blüten sind klein und bauchig. Dieser Typus kommt bei etwa einem Drittel der Streptocarpus-Arten vor, wobei viele Arten Selbstbestäuber sind. Von diesem ausgehend haben sich parallel die weiteren Blütentypen entwickelt.

Die überwiegende Zahl der Arten ist insektenblütig. Als Bestäuber kommen Bienen, Schmetterlinge und Fliegen (!) in Frage. Letzteres ist ungewöhnlich und einzigartig bei den Gesneriaceen. Der südafrikanische Biologe J. MANnING hat nach Mitteilung von Potgieter \& Edwards (2005) langrüsselige Fliegen (Stenobasipteron wiedemanni, Nemestrinidae) beim Blütenbesuch von Streptocarpus formosus beobachtet. Die Autoren DUB und MM konnten diese Bestäubung für Streptocarpus primulifolius bestätigen. Da die Blütenform dieser Arten vielen anderen Arten der Untergattung Streptocarpus ähnelt, muss man annehmen, dass hier Fliegenbestäubung eine große Rolle spielt.

Als typisch tropisches Phänomen kann die Bestäubung durch Wirbeltiere angesehen werden. Während bei den Gesneriaceen weltweit keine Fälle von Bestäubung durch nicht-fliegende Säugetiere (Therophilie) bekannt sind, spielt Vogelblütigkeit (Ornithophilie) in Mittel- und Südamerika durch die Existenz der Kolibris (Trochilidae) eine eminent wichtige Rolle. Dort und nur dort sind auch viele Fälle von Fledermausblütigkeit (Chiropterophilie) bekannt. In Asien gibt es nach bisherigem Wissen keine chiropterophilen Vertreter, und die Ornithophilie ist auf zwei große Gattungen, Aeschynanthus (ca. 180 Arten) und Agalmyla (ca. 100 Arten) konzentriert. Daneben gibt es ei-

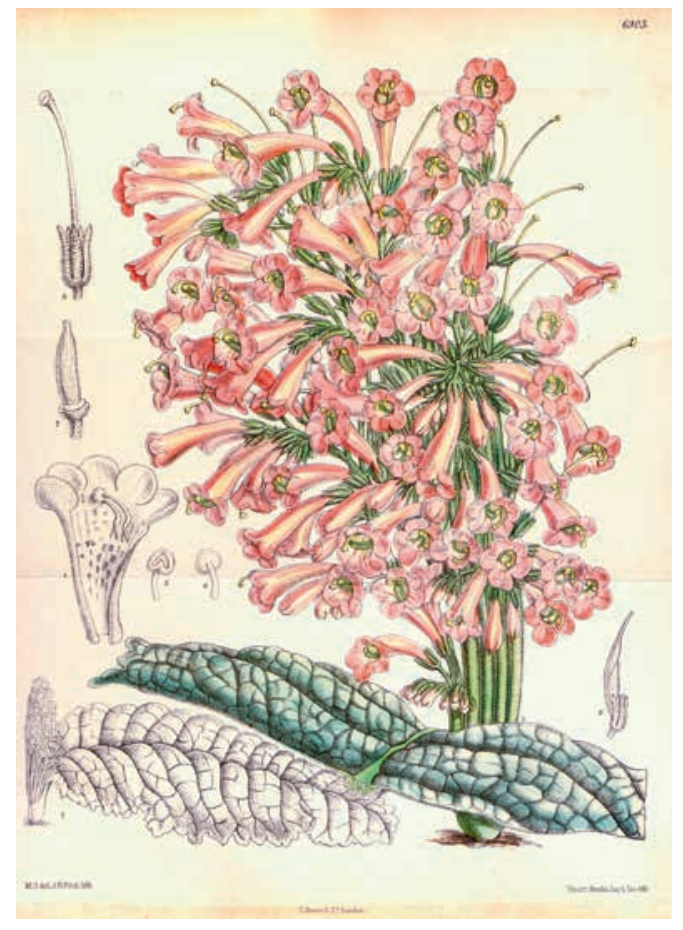

Abb. 5: Streptocarpus dunnii. (Aus J. D. Hooker 1886, T. 6903)

nige wenige ornithophile „Ausreißer“ in primär insektenblütigen Gattungen (z. B. Petrocodon coccineus als einzige vogelblütige Art von Petrocodon, 45 Arten). Vielleicht fallen auch einige Oreocharisund Didymocarpus-Arten mit orange-roten Blüten in diese Kategorie.

\section{Streptocarpus dunnii}

Im Gegensatz zu Streptocarpus myoporoides ist $S$. dunnii eine schon lange und gut bekannte Art. Sie wurde 1886 von J. D. Hooker in „Curtis's Botanical Magazine“ eingehend beschrieben und abgebildet (Abb. 5). Der Artbeiname dunnii ehrt den Geologen und Pflanzensammler E. G. DunN, der die Pflanze im damaligen Transvaal (heute Mpumalanga, eine nordöstliche Provinz der Republik Südafrika) entdeckte und Samen nach Kew schickte. Die Beschreibung und Abbildung HooKERs erfolgte nach einem kultivierten Exemplar.

Hooker war von der Pflanze sichtlich begeistert und spricht von einer „magnificent plant which is quite the monarch in its beautiful genus“. 


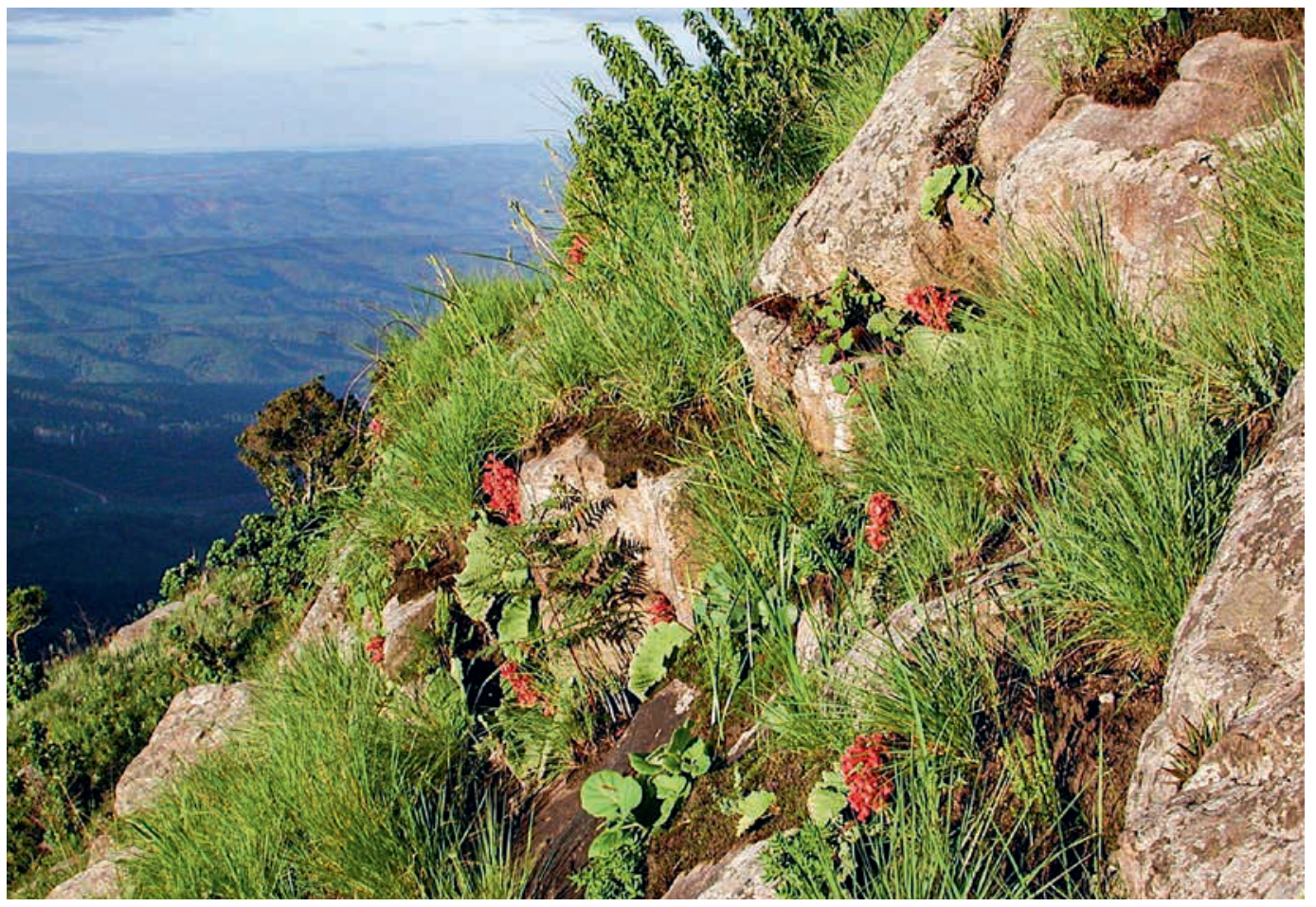

Abb. 6a: Streptocarpus dunnii, sterile und blühende Pflanzen in ihrem natürlichen Lebensraum in Mpumalanga, Südafrika: Steilhänge der Inland-Hochebene (ca. 2000 m), die auf die Tiefebene des Krüger Nationalparks abfallen. (Foto: A. Louw)

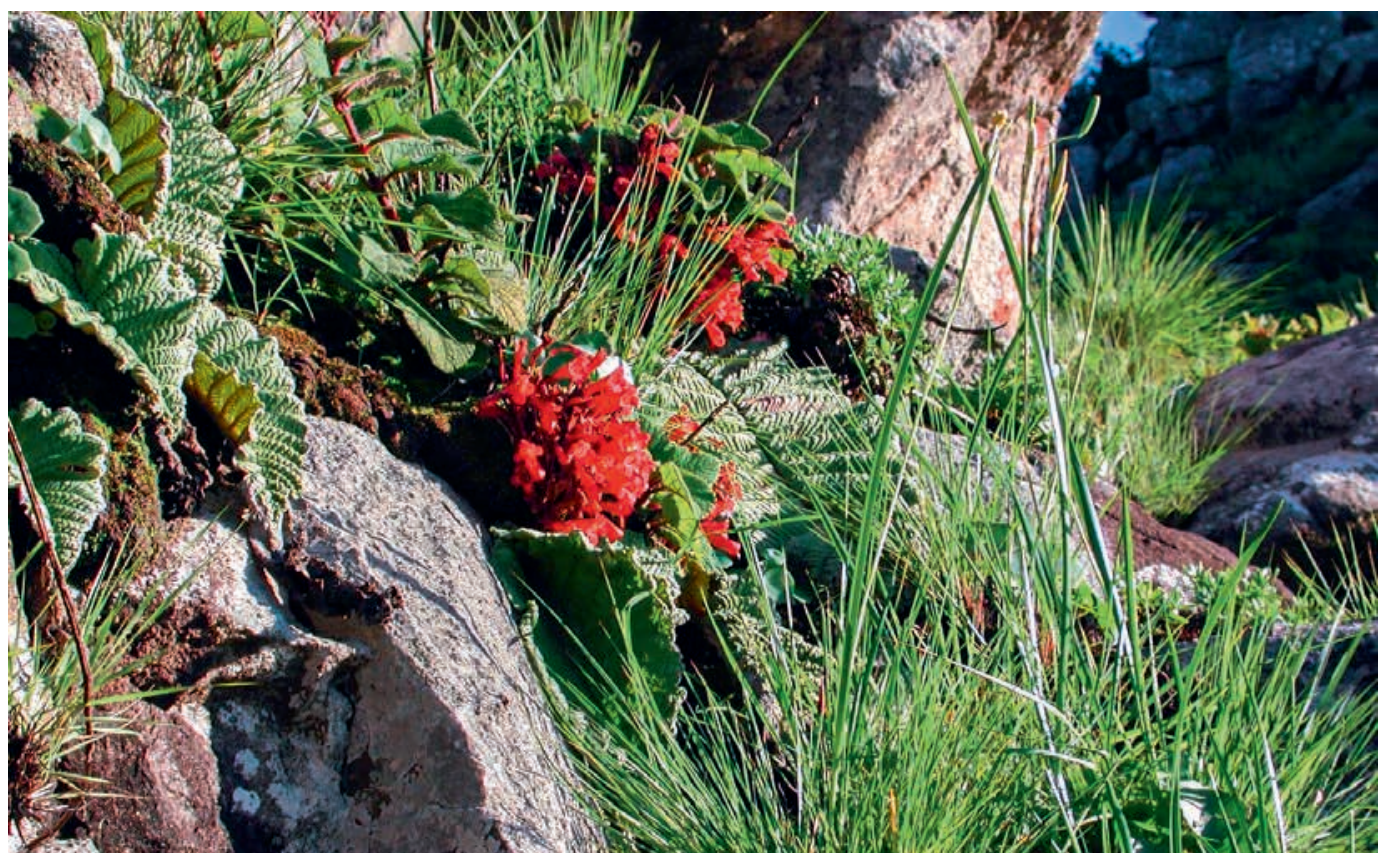

Abb. 6b: Streptocarpus dunnii (Mpumalanga, Südafrika). In der Begleitvegetation dominieren Gräser, der Vegetationskomplex wird daher als „Grasveld“ bezeichnet. (Foto: A. Louw) 


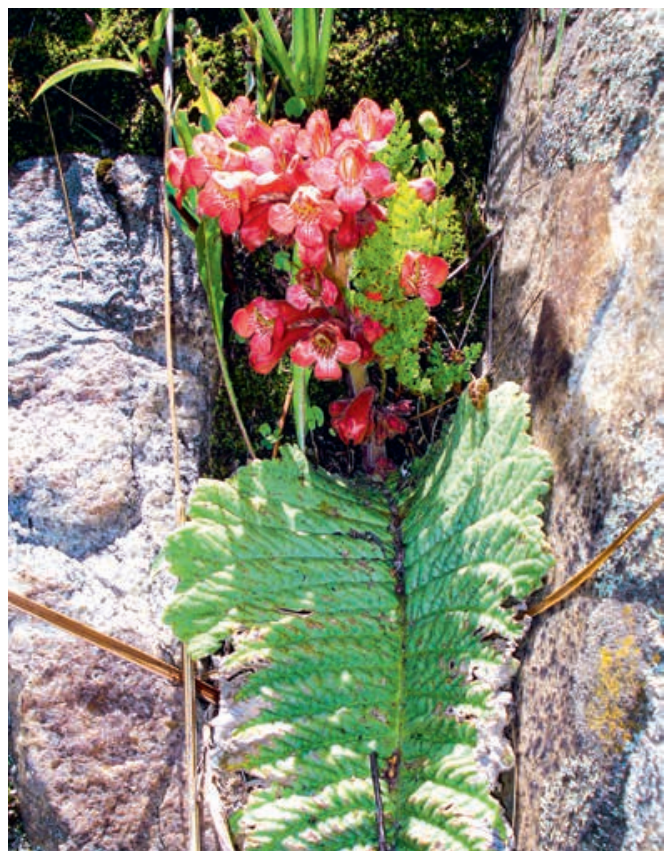

Abb. 7: Streptocarpus dunnii, blühende Einzelpflanze in ihrem natürlichen Habitat (Mpumalanga, Südafrika). Der Vegetationskörper umfasst nur ein einziges Blatt: ein Keimblatt, das bis zum Blühen und Fruchten der Pflanze weiterwächst (Makrokotyledo). (Foto: J. K. Welwitch)

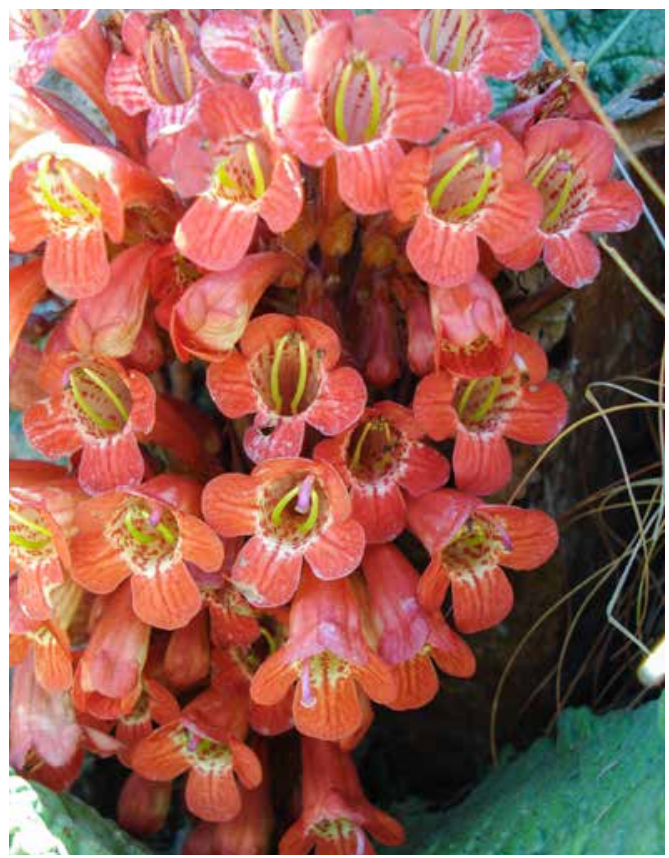

Abb. 8: Streptocarpus dunnii, Ausschnitt aus einem reichblütigen Blütenstand. (Foto: J. K. Welwitch)
Auch der mit Hooker befreundete Botaniker M. T. Masters, der in der Botanisch-Gärtnerischen Wochenzeitschrift „The Gardener's Chronicle“ (1886: 625) Werbung für Hookers Darstellung und das „Botanical Magazine“ machen wollte, sagt: „This is the truly extraordinary and by no means unattractive plant" und "We repeat that this is one of the most extraordinary plants that have ever been figured in that repertory of wonders and beauties, the Botanical Magazine“.

Das kleine Verbreitungsgebiet von $S$. dunnii nimmt einen zentralen Teil der Provinz Mpumalanga ein und reicht noch etwas in das Königreich Eswatini (früher Swasiland) hinein. Es ist dies ein Teil des Drakensberg-Zuges, der die östliche Begrenzung des südafrikanischen Zentral-Plateaus bildet.

Streptocarpus dunnii wächst in steilem und offenem Berggelände, ohne Bedeckung durch Bäume oder Sträucher, sodass die Pflanzen zeitweilig voll der Sonne ausgesetzt sind (Abb. 6a, b). Die dichten Blütenstände sind weithin sichtbar. Die wurzelnden Bereiche und Teile des einzelnen Blattes sind in Felsspalten verborgen, wo sich Humus und Feuchtigkeit halten können.

Streptocarpus dunnii gehört in die morphoökologische Gruppe der unifoliaten Streptocarpen. Der Vegetationskörper umfasst nur ein einziges Blatt, welches eines der beiden Keimblätter („Makrokotyledo“) ist (Abb. 7). Die Blütenstände entspringen zu mehreren an der Basis dieses Blattes in serial-absteigender Weise. Durch reiche Verzweigung, relativ dichte Anordnung und das fast gleichzeitige Aufblühen der Blüten entstehen große prächtige Büschel von Blüten (Abb. 7, 8). Die Pflanzen sind, wie HilliARD \& BuRTT (1971: 212) schreiben „even more strikingly beautiful when seen against the grey rocks of its native habitat". Biologisch gesehen: sie fallen schon aus weiter Entfernung auf und es ist klar, dass sie der visuellen Fernanlockung von Bestäubern dienen.

Wie schon Hooker festgestellt hat, variiert die Blütenfarbe von einem zarten Rosa zu einem tiefen 
Ziegelrot. Im Vergleich zu S. rexii, S. primulifolius etc. sind die Blüten von $S$. dunnii wesentlich kleiner, stärker röhrig und stabiler gebaut, die Kronzipfel sind kleiner und weniger ausladend. Die Blüten sind leicht gekrümmt und so positioniert, dass die Kronröhre in etwa waagerecht zu liegen kommt oder leicht nach unten zeigt. Die Staubblätter sind stark aufwärts gekrümmt, wodurch die beiden verbundenen Antheren oben am Eingang des Kronschlundes liegen. In Farbe, Form und funktioneller Konstruktion sind die Blüten wie geschaffen für die Bestäubung durch Vögel.

\section{Streptocarpus myoporoides}

Streptocarpus myoporoides wurde erst 1968 von Hilliard \& BurtT beschrieben, wobei der Name in folgender Form erschien: Streptocarpus (?) myoporoides. Das Fragezeichen setzten die Autoren deswegen, weil der einzige Beleg, den sie damals zur Verfügung hatten, keine Früchte hatte und daher eine finale Gattungszuordnung nicht möglich war. Später, in Hilliard \& BurTt (1971), konnte das Fragezeichen entfernt werden, weil sich in einer inzwischen getätigten weiteren Aufsammlung auch Früchte fanden. Sie waren gedreht und somit konnte die Art definitiv zu Streptocarpus (s. s.) zugeordnet werden.

Die Verbreitung von S. myoporoides ist sehr begrenzt, sogar stärker als für $S$. dunnii. Die Pflanze ist ein Lokalendemit der Ribáuè und Chinga Gebirge im Norden Mosambiks. Die wenigen bisherigen Aufsammlungen stammen von diesen Bergmassiven, die hier publizierten Fotos sind von Ton Rulkens in ersterem Gebirge aufgenommen worden.

Ähnlich wie $S$. dunnii wächst auch $S$. myporoides auf steilem, $z$. T. senkrecht abfallenden Gelände (Abb. 9). Das Terrain ist aber bewaldet und schattig (montaner Regenwald in ca. $1500 \mathrm{~m}$ Höhe). Der Untergrund ist felsig (Granit), permanent feucht und von Moosen und Selaginellen bewachsen.

Schon auf den ersten Blick kann man erkennen, dass der Vegetationskörper anders gebaut ist als bei $S$. dunnii. Neben einem großen Blatt, sicht-

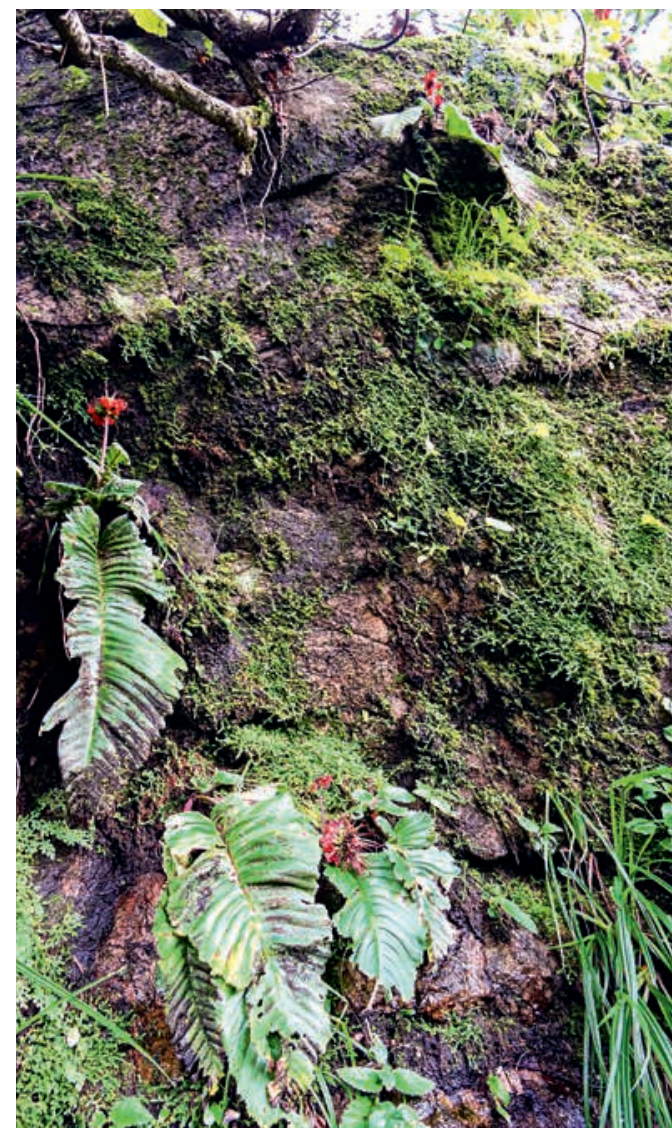

Abb. 9: Streptocarpus myoporoides, blühende Pflanzen in ihrem natürlichen Habitat im Mt. Ribáue National Park, Mosambik. (Foto: T. Rulkens)

lich der Makrokotyledo (Mk), gibt es noch weitere Blätter, zum Teil kleiner als der Mk, z. T. aber die gleiche Größe erreichend (Abb. 10). Die auf den Mk folgenden ersten Blätter sind gegenständig, später scheint die Wirtelstellung aufgelöst zu werden. Die Anordnung der Blätter ist nicht wie bei den meisten rosulaten Streptocarpus-Arten in einer zentrischen oder exzentrischen Rosette, sondern, soweit man das aus den Fotos schließen kann, unregelmäßig.

Bei den Infloreszenzen fällt auf, dass ein langer Stiel und ein terminaler Blütenschopf vorhanden sind und damit eine Ähnlichkeit zum asiatischen Petrocodon coccineus besteht (Parallelevolution). Die Blüten sind scharlachrot und neigen sich leicht nach unten. Sie blühen von innen (Infloreszenz- 

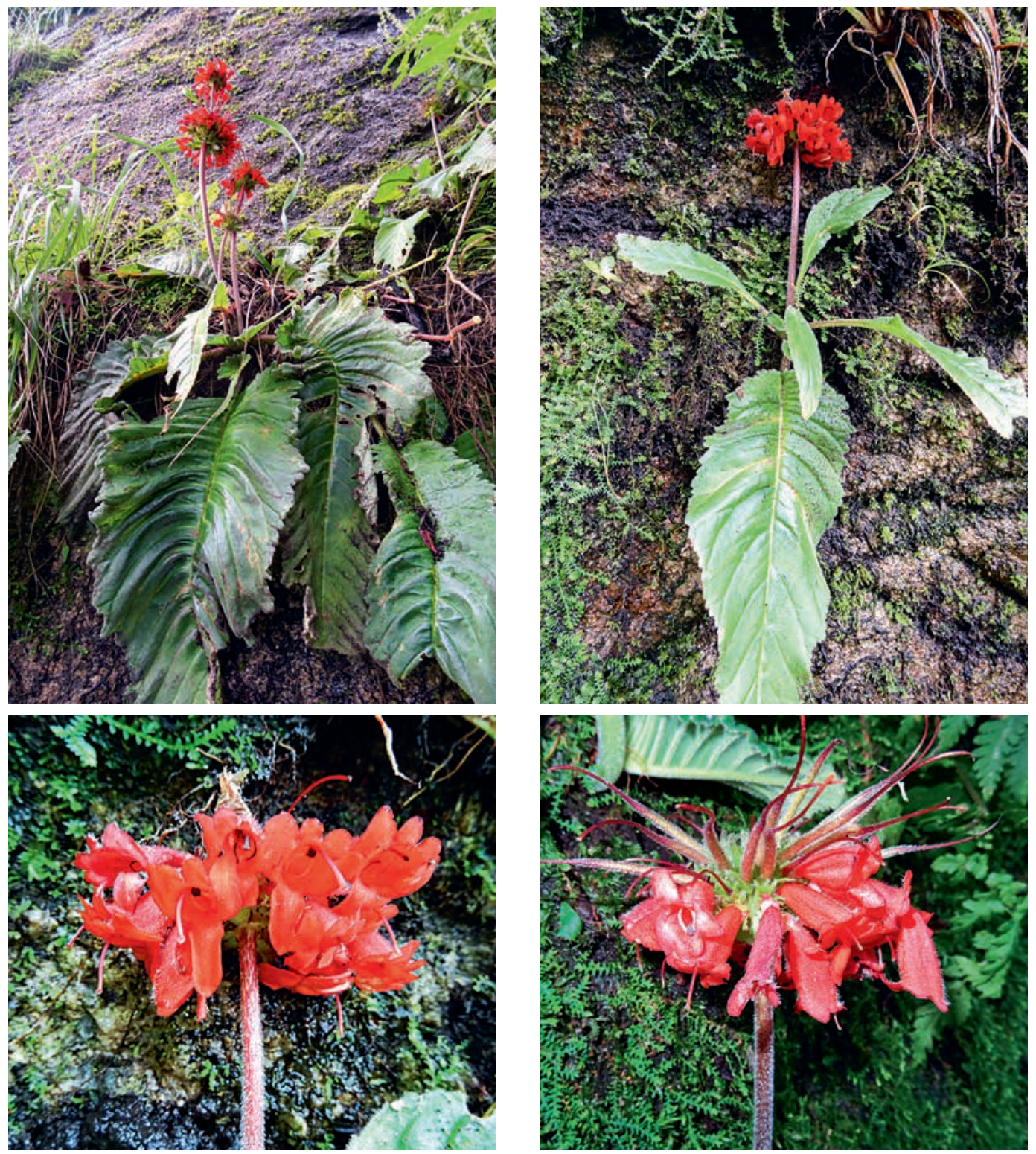

Abb. 10: Streptocarpus myoporoides im Ribáuè National Park, Mosambik. Oben: Blühende Pflanzen in ihrem natürlichen Habitat. Unten: Infloreszenz im voll blühenden und (rechts) teilweise fruchtenden Zustand. (Foto: T. RuLKens)

zentrum) nach außen auf (Abb. 10). Die Fruchtbildung und -reifung erfolgt in der gleichen Weise.

Auffällig ist auch die Differenz zu $S$. dunnii im Blütenbau. Die Krone ist zweilippig, aber in einer Weise, wie sie sonst nirgends sonst bei den afrikanischen Gesneriaceen anzutreffen ist. Die Oberlippe wird von vier (anstelle von zwei)
Kronzipfeln gebildet, während die Unterlippe normalerweise dreilappig - nur aus einem Zipfel besteht (Abb. 10, links unten). Solche Kronenformen sind bei vielen vogelblütigen Taxa der Neotropen (z. B. Columnea) und Asiens (z. B. Petrocodon coccineus, siehe oben) zu finden. Der funktionelle Hintergrund: Die vergrößerte Oberlippe bildet einen dachartigen Vorsprung, unter 


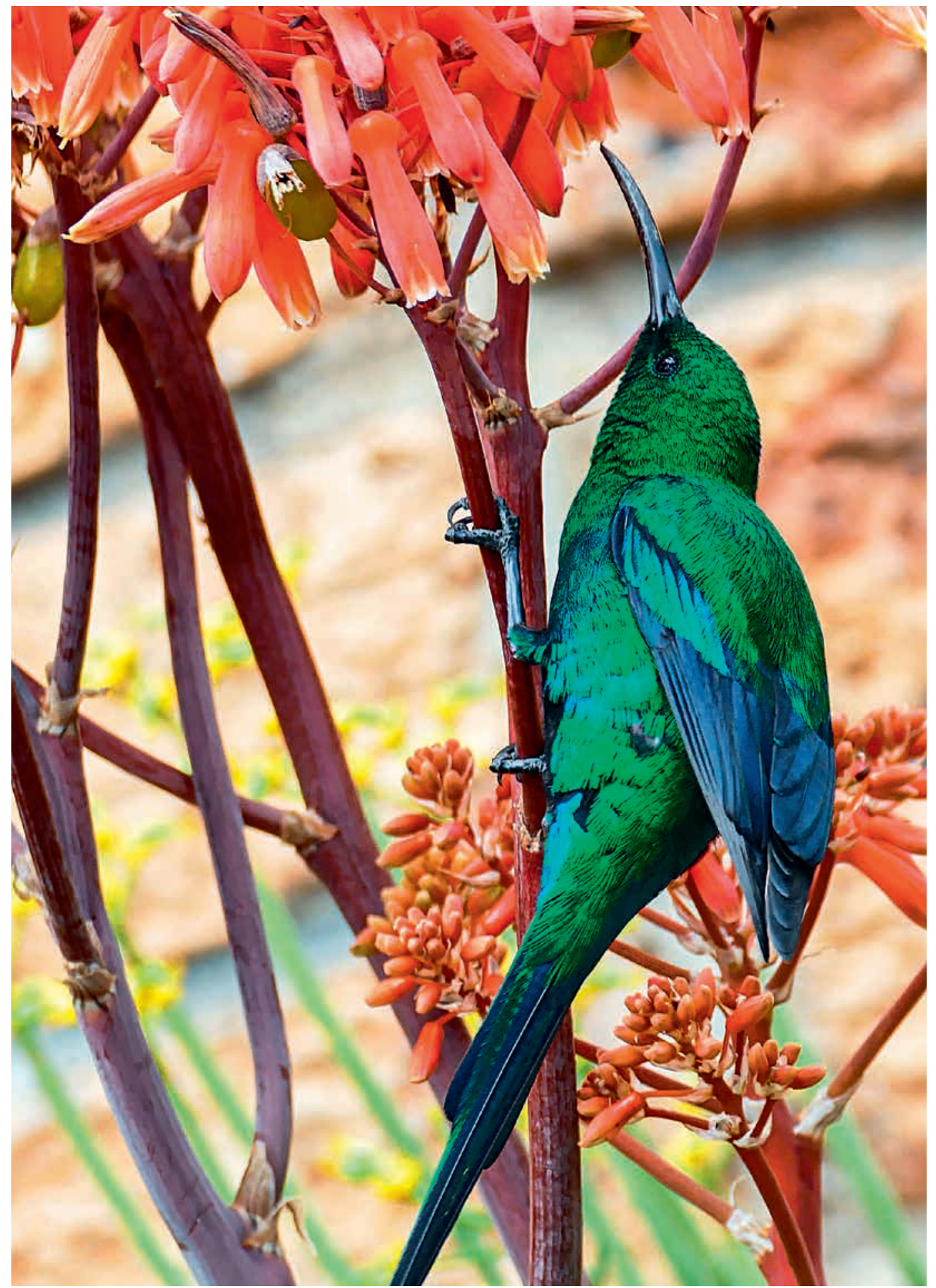

Abb. 11: Malachit-Nektarvogel (Nectarinia formosa, Männchen) an den Blüten der Korallen-Aloe (Aloe stricta); Rest Camp, Mountain Zebra National Park, Eastern Cape Prov., Südafrika. (Foto: B. Dupont, Wikipedia, Lizenz: CC BY 2.0 ) 
dem die Antheren geschützt liegen. Der Vogel kommt mit der Oberseite des Schnabels oder der Stirn mit den Antheren in Berührung (nototribe Pollenbelegung). Doch nichts ist ohne Ausnahme: In Asien gibt es auch klar insektenblütige Arten, die einen ähnlichen Kronenbau aufweisen (z. B. Allocheilos; sehr schön dokumentiert bei den kürzlich neubeschriebenen Arten A. maguanensis und A. rubroglandulosus, Chen et al. 2020) und Oreocharis mileensis (MöLler et al. 2011).

Trotz der verschiedenen Lippenkonstruktion bei $S$. dunnii und $S$. myoporoides ist die äußere Form der Blüte sehr ähnlich. Die Krone ist robust, röhrig, mit leichter und kontinuierlicher Erweiterung gegen den Schlund zu, und die Kronzipfel sind relativ klein und nicht ausgebreitet. Es ist dies die funktionelle Form einer Vogelblüte. Soweit man auf den Fotos sehen kann, blühen die Blüten auf, wenn sie etwa eine horizontale Position erreicht haben und neigen sich dann langsam nach unten. Da die Nektarund Honigvögel nicht im Schwirrflug, sondern die Blüten im Sitzen ausbeuten (siehe unten), muss ihnen auch ein Sitzplatz in der Nähe der Blüten bereitgestellt werden. Das ist bei $S$. dunnii der Makrokotyledo, bei $S$. myoporoides der lange, robuste Infloreszenzstiel.

\section{Nektar- und Honigvögel als mögliche Bestäuber von Streptocarpus dunnii und S. myoporoides}

Der erste, der die Vermutung aussprach, dass $S$. dunnii ornithophil sei, war Stefan Vogel in seinem Buch „Blütenbiologische Typen als Elemente der Sippengliederung: dargestellt anhand der Flora Südafrikas" (Vogel 1954, siehe auch Hilliard \& Burtt 1971). Konkrete Beobachtungen konnte er nicht machen.

Als blütenbesuchende Vögel sind im südlichen Afrika in erster Linie die Nektar- und Honigvögel von Bedeutung.

Die Nektarvögel (Nectarinidae) bilden eine Familie, die etwa 145 Arten in 15 Gattungen enthält und deren Verbreitungsgebiet sich vom westlichen Afrika bis nach Australien erstreckt.

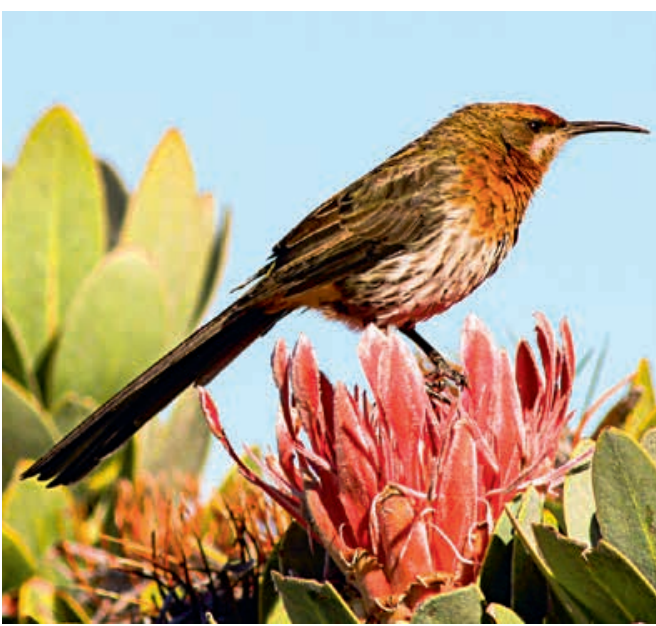

Abb. 12. Ein Natal-Honigvogel (Promerops gurneyi) auf dem Blütenstand eines Silber-Zuckerbusches (Protea roupelliae). Marakele National Park, Prov. Limpopo, Südafrika.

(Foto: D. Keats, Wikipedia, Lizenz: CC BY 2.0)

In Südafrika gibt es 21 Arten, die sich auf 7 Gattungen verteilen. Charakteristisch sind der lange, gebogene Schnabel und die noch längere, röhrenförmige Zunge, die am Ende quastenförmig aufgespalten ist. Sie kann bis zur doppelten Länge des Schnabels ausgestreckt werden und ist daher hervorragend eignet, den Nektar aus Blüten zu entnehmen.

Die Honigvögel (Promeropidae) enthalten nur eine Gattung (Promerops) mit zwei Arten. Ihre Verbreitung ist auf das südliche bzw. südöstliche Afrika beschränkt. Für Streptocarpus dunnii wie auch $S$. myoporoides kommt nur eine Art, der Natal-Honigvogel (Promerops gurneyi, Abb. 12), als potenzieller Bestäuber in Frage, da nur dieser im Verbreitungsgebiet der beiden Pflanzen vorkommt.

\section{Bestäubungsbeobachtungen}

Beobachtungen fehlten bis vor kurzem, aber es gibt jetzt einen aktuellen Hinweis: MöLLER et al. (2019) berichten, dass Bellstedt, Hughes \& Möller (unveröff.) den Blütenbesuch eines Malachit-Nektarvogels (Nectarinia formosa, Abb. 11) an Streptocarpus dunnii beobachten konnten. Leider gibt es davon keine Dokumentationsfotos. 
Wie nach der Blütenkonstruktion zu erwarten, sitzt der Vogel zur Nektarentnahme auf dem Makrokotyledo. Währenddessen findet die Pollendeposition an der Stirn des Vogels (nototrib) statt. Diese Blütenbesuche dauern nur wenige Sekunden.

Was Streptocarpus myoporoides betrifft, gibt es bisher keine Beobachtungen und solche dürften auf Grund des entlegenen Standorts auch schwierig sein. Als potenzielle Bestäuber kommen der Malachit-Nektarvogel (Nectarinia formosa), der Bronze-Nektarvogel ( $N$. kilimensis) und der schon erwähnte Natal-Honigvogel (Promerops gurneyi) in Frage.

\section{Phylogenetische Schlussfolgerungen}

Hilliard \& Burtt (1971) lassen in ihren Artenbeschreibungen $S$. dunnii und $S$. myoporoides unmittelbar aufeinanderfolgen, wohl in der Annahme, dass die beiden eng miteinander verwandt sind. Auf Grund ihrer Unterschiede ist das allerdings nicht sehr wahrscheinlich. Leider existieren molekulare Daten zur Zeit nur für $S$. dunnii und diese besagen, dass diese Art zu Streptocarpus subg. Streptocarpus sect. Streptocarpus gehört (Nishi et al. 2015). Dies mag auf Grund des vegetativen Aufbaus auch für $S$. myoporoides zutreffen. Das besagt aber nicht, dass die beiden Arten eng verwandt sind und die Ornithophilie in der Stammesgeschichte nur einmal entstanden ist. Die Autoren dieses Beitrages halten es für wahrscheinlicher, dass die Ornithophilie zweimal voneinander unabhängig und an verschiedenen Orten entstanden ist. Ob dies tatsächlich zutrifft, müssen zukünftige molekular-phylogenetische Untersuchungen zeigen.

\section{Warum ist Ornithophilie bei den afrikanischen Gesneriaceen so selten?}

Diese Frage lässt sich zur Zeit nur unbefriedigend und spekulativ beantworten. Eine Rolle spielt sicher die Tatsache, dass in Süd- und Mittelamerika eine ungleich höhere Zahl an Vogelbestäubern zur Verfügung steht. Die Kolibris (mit etwa 360 Arten) sind zudem kleine, akrobatische Flieger und ernähren sich fast ausschließlich von Nektar. Vergleicht man Südafrika, so sind nur etwas mehr als 20 Vogelarten in das Bestäubungsgeschehen ein- gebunden. Die Nektar- und Honigvögel trinken nicht nur Nektar, sondern ernähren sich auch von Insekten, sind also in geringerem Grad an Blüten gebunden. Ein weiterer Grund ist, dass es bei den afrikanischen Gesneriaceen kaum Epiphyten gibt, wie sie z. B. im tropischen Amerika etwa 40 Prozent der Gesneriaceenflora ausmachen (WiEHLER 1983). Epiphyten kommen den Bestäubern besser entgegen, als Pflanzen, die am oder knapp über dem Boden auf Felsen wachsen.

Es herrschen somit in Afrika sowohl von den Pflanzen, den Bestäubern, den edapischen und klimatischen Bedingungen her ganz andere Verhältnisse als in anderen Teilen der Tropen und Subtropen der Erde. Zusammengenommen gilt hier wohl die schon zitierte Phrase: „Africa - odd man out".

\section{Dank}

Die Ergebnisse dieses Betrages sind nur möglich geworden, weil in den letzten Jahren Biologen und Amateur-Naturforscher tolle Fotos aufgenommen und den Autoren zur Verfügung gestellt haben. Das sind in alphabetischer Reihenfolge der Familiennamen: Aart Louw, Ton Rulkens, und Judd Kirkel Welwitch. Ihnen sei an dieser Stelle herzlich gedankt.

\section{Literatur}

Bransgrove, K. \& Middleton, D. J. 2015: A revision of Epithema. - Gardens' Bull. Singapore 67: 159-229.

Chen, W. H., Guo, S. W., Wu, J. Y., Chen, L. \& Shui, Y. M. 2020: Two new species of Allocheilos (Gesneriaceae) from the karst regions in Yunnan, China. - PhytoKeys 157: 155-166.

Christenhusz, M. 2012: On African violets and Cape primroses - Towards a monophyletic Streptocarpus (Gesneriaceae). Phytotaxa 46: 3-9.

Darbyshire, I. 2006: Gesneriaceae. In: Beentje, H. J. \& S. A. Ghazanfar (eds.): Flora of tropical East Africa. - Royal Botanic Gardens, Kew.

Harrison, C. J., Möller, M. \& Cronk, Q. C. B. 1999: Evolution and development of floral diversity in Streptocarpus and Saintpaulia. - Ann. Bot. 84: 49-60. 
Hilliard, O. M. \& Burtt, B. L. 1968: Studies in the Gesneriaceae of the Old World XXVII: New species and subspecies of Streptocarpus. - Notes Roy. Bot. Gard. Edinburgh 28: 209-215.

Hilliard, O. M. \& Burtt, B. L. 1971: Streptocarpus - An African plant study. - University of Natal Press, Pietermaritzburg.

Hooker, J. D. 1886: Streptocarpus dunnii. Native of the Transvaal. - Curtis's Bot. Mag. 112: T. 6903.

Hooker, W. J. 1827: Didymocarpus rexii. Twisted-fruited Didymocarpus - Exot. Fl. 3: 227-228, T. 227.

Hughes, M., MacMaster, G., Möller, M., Bellstedt, D. U. \& Edwards, T. J. 2006: Breeding system of a plesiomorphic floral type: An investigation of small flowered Streptocarpus (Gesneriaceae) species. - Pl. Syst. Evol. 262: 13-24.

Lindley, J. 1828: Streptocarpus rexii. Cape Streptocarpus. Bot. Reg. 14: T. 1173 (1828).

Möller, M. \& Cronk, Q. C. B. 1997a: Origin and relationships of Saintpaulia (Gesneriaceae) based on ribosomal DNA internal transcribed spacer (ITS) sequences. - Amer. J. Bot. 84: 956-965.

Möller, M. \& Cronk, Q. C. B. 1997b: Phylogeny and disjunct distribution: Evolution of Saintpaulia (Gesneriaceae). - Proc. Roy. Soc. London B 264: 1827-1836.

Möller, M. \& Cronk, Q. C. B. 1999: New approaches to the systematics of Saintpaulia and Streptocarpus. In: ANDREws, S., Leslie, A. \& Alexander, C. (eds.): Taxonomy of cultivated Plants. - Third Int. Sympos. Kew, Kew.

Möller, M. \& Cronk, Q. C. B. 2001: Phylogenetic studies in Streptocarpus (Gesneriaceae): Reconstruction of biogeographic history and distribution patterns. - Syst. Geogr. Pl. 71: 545-555.

Möller, M., Middleton, D. J., Nishit, K., Wei, Y. G., Sontag, S. \& Weber, A. 2011: A new delineation for Oreocharis incorporating an additional ten genera of Chinese Gesneriaceae. - Phytotaxa 23: 1-36.

Möller, M., Barber, S., Atrins, H. J. \& Purvis, D. A. 2019: The living collection at the Royal Botanic Garden Edinburgh illustrates the floral diversity in Streptocarpus (Gesneriaceae). - Sibbaldia 17: 155-175.

Nishit, K., Hughes, M., Briggs, M., Haston, E., Christie, F., DeVilliers, M. J., Hanekom, T., Roos, W. G., Bellstedt, D. \& Möller, M. 2015: Streptocarpus redefined to include all Afro-Malagasy Gesneriaceae: Molecular phylogenies prove congruent with geographical distribution and basic chromosome numbers and uncover remarkable morphological homoplasies. - Taxon 64: 1243-1274.

Potgieter, C.J. \& Edwards, T. J. 2005. The Stenobasipteron wiedemanni (Diptera, Nemestrinidae) pollination guild in eastern southern Africa. - Ann. Missouri Bot. Gard. 92: 254-267.

Shorthouse D.P. 2010. SimpleMappr, an online tool to produce publication-quality point maps. [Retrieved from https:// www.simplemappr.net. Accessed May 20, 2021].
Vogel, S. 1954: Blütenbiologische Typen als Elemente der Sippengliederung, dargestellt anhand der Flora Südafrikas. Bot. Studien, Heft 1: 1-338. Jena.

Weber, A., Clark, J. L. \& Möller, M. 2013: A new formal classification of Gesneriaceae. - Selbyana 31: 68-94.

Wiehler, H. 1983: A synopsis of Neotropical Gesneriaceae.Selbyana 6: 1-219.

\section{Internetseiten}

Liste der Vögel Afrikas: 85 Arten; https://en.wikipedia.org/ wiki/List_of_birds_of_Africa

Liste der Vögel des südlichen Afrika: 21 Arten; https://en.wikipedia.org/wiki/List_of_birds_of_Southern_Africa

Honigvögel: https://de.wikipedia.org/wiki/Honigv\%C3 $\%$ B6gel

Natal-Honigvogel: https:/en.wikipedia.org/wiki/Sugarbird\#/ media/File:Gurney's_Sugarbird,_Promerops_gurneyi_at_Marakele_National_Park_(14142942643).jpg

Nektarvögel: https://de.wikipedia.org/wiki/Nektarv\%C3\% B6gel

Malachit-Nektarvogel: https://upload.wikimedia.org/wikipe $\mathrm{dia} /$ commons/thumb/0/0c/Malachite_Sunbird_\%28Necta rinia_famosa\%29_male_on_Aloe_flowers_\%2830216015541 $\% 29 . j p g / 3648$ px-Malachite_Sunbird_\%28Nectarinia_famosa\%29_male_on_Aloe_flowers_\%2830216015541\%29.jpg

\section{Anschrift der Autoren}

Prof. i. R. Dr. Anton Weber, Department für Botanik und Biodiversitätsforschung, Universität Wien, Rennweg 14, A-1013 Wien. E-Mail: anton.weber@univie.ac.at

Prof. Dr. Dirk U. Bellstedt, Biochemistry Department, Stellenbosch University, South Africa.

E-Mail: dub@sun.ac.za

Dr. Michael Möller, Royal Botanic Garden Edinburgh, Scotland, U.K. E-Mail: MMoeller@rbge.org.uk 